\title{
Position-dependent effects of cytosine methylation on FWA expression in Arabidopsis thaliana
}

\author{
Thanvi Srikant', Anjar Wibowo ${ }^{1,2}$, Rebecca Schwab' and Detlef Weigel' \\ 'Max Planck Institute for Developmental Biology, Max-Planck-Ring 9, 72076 Tübingen, Germany \\ ${ }^{2}$ Faculty of Science and Technology, Airlangga University, Kampus C, Mulyorejo, Surabaya City, East Java \\ 60115, Indonesia
}

\begin{abstract}
Gene expression can be modulated by epigenetic modifications to chromatin, and variants of the same locus distinguished by fixed, heritable epigenetic differences are known as epialleles. DNA methylation at cytosines is a prominent epigenetic modification, particularly in plant genomes, that can modulate gene expression. There are several examples where epialleles are associated with differentially methylated regions that affect the expression of overlapping or close-by genes. However, there are also many differentially methylated regions that have not been assigned a biological function despite their proximity to genes. We investigated the positional importance of DNA methylation at the FWA (FLOWERING WAGENINGEN) locus in Arabidopsis thaliana, a paradigm for stable epialleles. We show that cytosine methylation can be established not only over the well-characterized SINE-derived repeat elements that overlap with the transcription start site, but also in more distal promoter regions. FWA silencing, however, is most effective when methylation covers the transcription start site.
\end{abstract}




\section{INTRODUCTION}

Methylation of cytosine nucleotides in DNA is a prominent epigenetic mark in plant and animal genomes (I). It is found mostly over transposons and repeat elements, consistent with its primary function in silencing their transcriptional activity in association with methylation at lysine 9 of histone 3 (H3K9) (2). Mutants with defects in METHYLTRANSFERASE I (METI), encoding the major methyltransferase maintaining cytosine methylation in Arabidopsis thaliana, display various phenotypic abnormalities such as delayed flowering, dwarfism, and sterility, with increasing severity during successive rounds of inbreeding $(3,4)$. Genomes of metl mutants are largely hypomethylated at CG dinucleotides that usually inherit cytosine methylation faithfully during DNA replication through METI activity (5). Phenotypic abnormalities do, however, not require whole-genome changes in cytosine methylation, as a recent study describes how hypomethylation at a few select loci is sufficient to establish quantitative resistance to a pathogenic oomycete, Hyaloperonospora arabidopsidis (Hpa) (6).

Transposable elements and repeats in the $A$. thaliana genome are mostly confined to heterochromatic regions, such as pericentromeres and telomeres. Others are distributed in euchromatic regions, and their proximity to protein-coding genes has been associated with constitutive or induced silencing of these proximal genes upon changes in cytosine methylation $(7,8)$. There are several examples of loci with alternative states of cytosine methylation and associated gene expression; the variants are known as epialleles. One example comes from the SDC (SUPPRESSOR OF $d d c$ ) locus with a direct tandem repeat in its promoter. When methylation at the repeat is absent, SDC is expressed, resulting in a dwarfed phenotype (9). Epialleles can also form following stress exposure; treatment with the 22-amino-acid peptide flg22, an immune-response inducing fragment of the bacterial flagellin protein, causes differential methylation of helitron-derived repeats lying within a $3 \mathrm{~kb}$ promoter region of the defense gene RESISTANCE METHYLATED GENE I (RMGI), and ensuing activation of RMGI expression (10). Furthermore, cytosine methylation has been shown to modify gene expression when located farther away from the gene body, such as in the case of FLOWERING LOCUS T(FT), where methylation on two enhancers located $5 \mathrm{~kb}$ upstream and I kb downstream of the gene 
can repress transcriptional activity (II). This was shown by experimentally targeting cytosine methylation to these enhancers using Inverted Repeat-Hairpins (IR-Hairpins), which lead to the downregulation of $F T$ expression and delayed flowering.

While these examples provide substantial evidence for methylation-dependent transcriptional changes, the requirements for cytosine methylation to exert this function is not well understood, as not all cytosine methylation, even when densely focused in methylated regions, triggers silencing of adjacent genes (12).

To address such functional differences, we investigated the promoter of the well-characterized Arabidopsis thaliana fwa-I epiallele. The FWA (FLOWERING WAGENINGEN) locus (At4G25530) harbors two sets of tandem repeats originating from a SINE3 retrotransposon (I3). These repeats overlap the promoter and the FWA transcribed sequence, and are covered by dense CG methylation in wild-type plants. Throughout vegetative development, FWA is transcriptionally inactive, and activated only in the female gametophyte and endosperm by maternal imprinting, when DNA methylation is erased (14). Methylation at the repeats is also absent in fwa-I epimutants, where the gene is constitutively active, which results in late flowering (15-18).

It is known that the presence of cytosine methylation at a specific position, the SINE-derived repeat elements, imposes transcriptional silencing on the entire locus. We made use of the unmethylated promoter in the fwa-I epiallele and asked the reverse, whether FWA silencing can be triggered only by cytosine methylation at these repeats, or whether it can be similarly induced when cytosine methylation is artificially directed to other, non-repetitive, promoter elements. 


\section{RESULTS}

\section{Hairpins directing methylation to the FWA promoter}

We chose three regions of 100 or 200 basepairs (bp) in length, located approximately 100, 500 and 700 bp upstream of the FWA transcription start site (Table I and Figure I), and generated inverted repeat (IR)-hairpins (I9), intended to introduce cytosine methylation at these regions that are otherwise unmethylated in fwa- / mutants.

For each region, we generated two IR-hairpins, differing in the orientation of sense and antisense sequences within the hairpin (Table I, Figure I, Methods).

Table I : IR-Hairpin constructs targeting the FWA promoter

\begin{tabular}{|l|l|l|l|}
\hline Construct & Length (bp) & $\begin{array}{l}\text { Orientation of 5' arm target sequence } \\
\text { relative to FWA transcription }\end{array}$ & $\begin{array}{l}\text { Distance from FWA } \\
\text { transcription start } \\
\text { site }\end{array}$ \\
\hline IA & 100 & sense & $\begin{array}{l}-122 \text { to }-22 \\
\text { (Overlap with short } \\
\text { tandem repeats) }\end{array}$ \\
\hline IB & 100 & antisense & $\begin{array}{l}-122 \text { to }-22 \\
\text { (Overlap with short } \\
\text { tandem repeats) }\end{array}$ \\
\hline 2A & 100 & sense & -659 to -559 \\
\hline 2B & 100 & antisense & -659 to -559 \\
\hline 3A & 200 & sense & -936 to -736 \\
\hline 3B & 200 & antisense & -936 to -736 \\
\hline
\end{tabular}


A

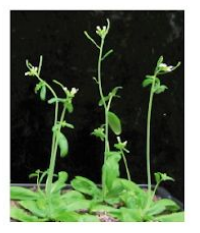

Ler wildtype

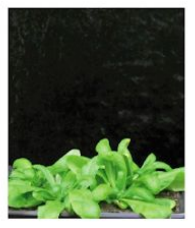

fwa-1 (Ler) epimutant

C

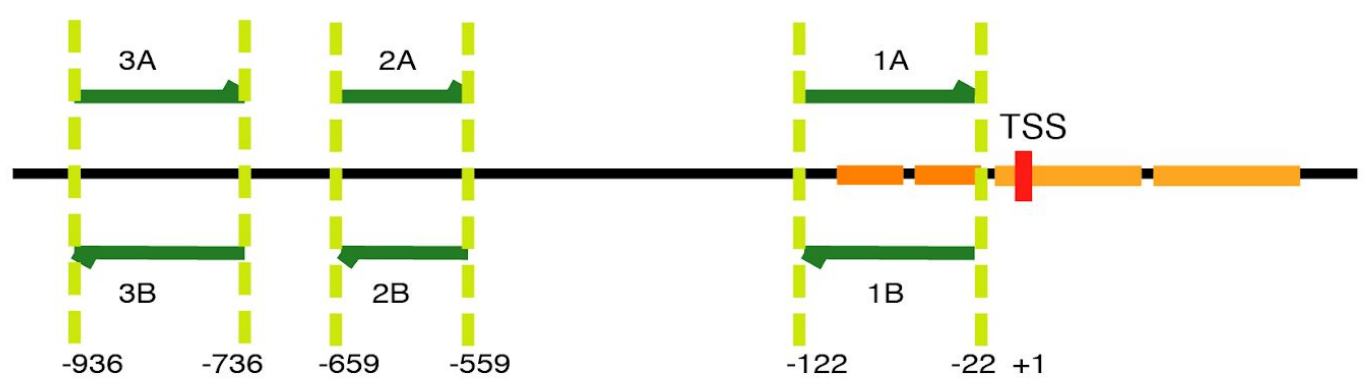

FWA (At4g25530)

C
B

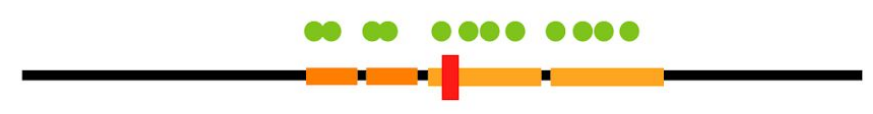

Tandem Repeats

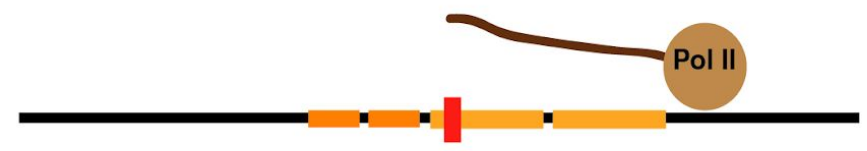

Tandem Repeats

Figure I. Methylation tiling in the FWA promoter

The diagram illustrates the FWA locus and the regions targeted for methylation tiling. (A) Late flowering phenotype of fwa-I (B) Release of methylation in the tandem repeats which overlap the TSS activates the FWA transcript (C) Three Regions in the FWA promoter chosen for hairpin-induced targeted methylation. Red line indicates the transcription start site (TSS) and the orange horizontal bars indicate the tandem repeats.

\section{Region-specific methylation effects on FWA activity}

We transformed late-flowering fwa-I mutants with IR-hairpin transgenes as described above (Table I), and identified homozygous insertion lines to monitor flowering time as a proxy for FWA activity. Corroborating previous work $(15-18,20)$, hairpins derived from sequences overlapping the repeat elements surrounding the transcription start site of FWA (lines IA and IB) accelerated flowering. This was different for hairpins derived from more distal promoter 
sequences (lines $2 \mathrm{~A}, 2 \mathrm{~B}, 3 \mathrm{~A}$ and $3 \mathrm{~B}$ ), where we did not observe obvious flowering differences compared to parental fwa- $/$ individuals (Figure 2 and Figure 3).

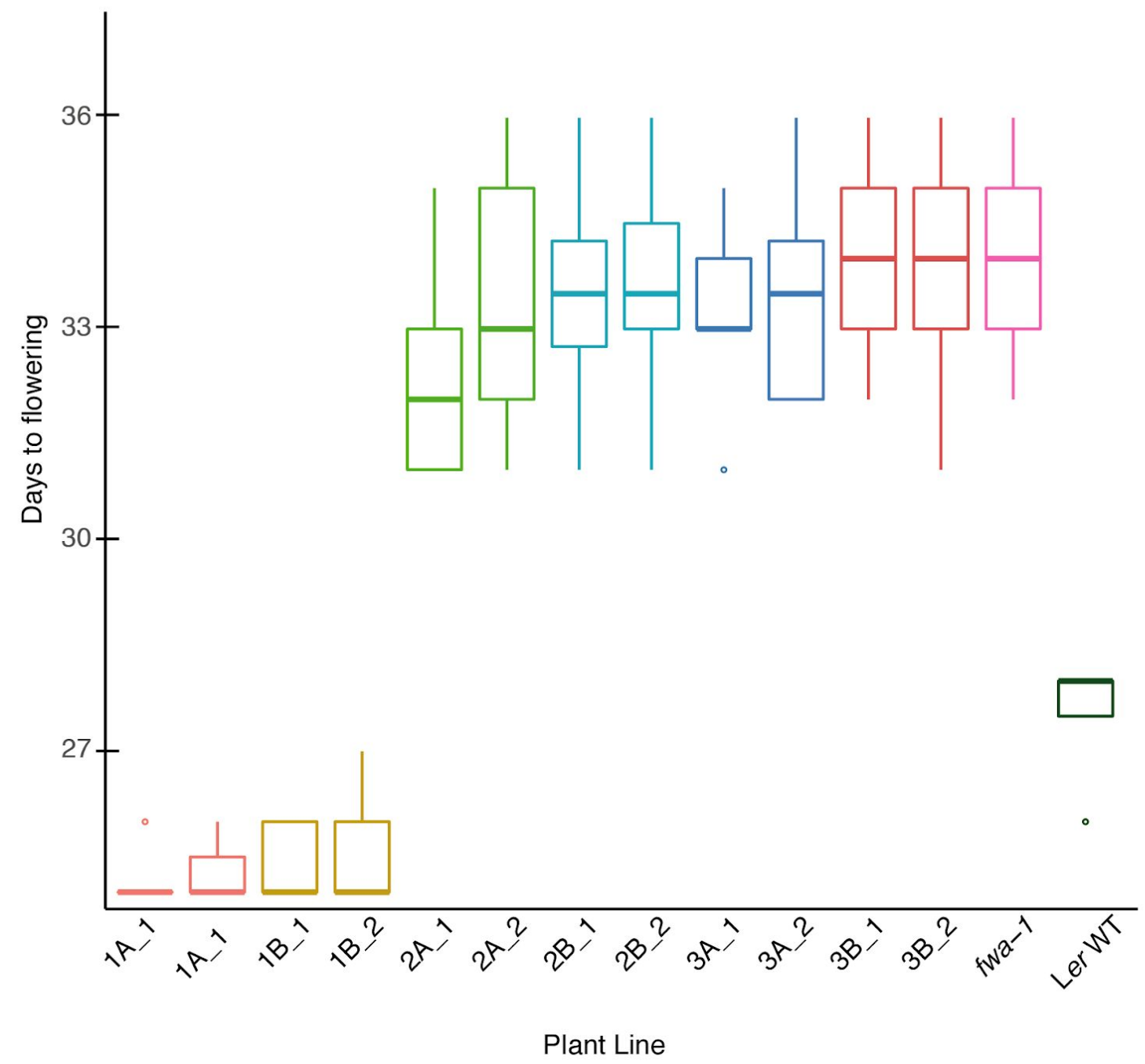

Figure 2. Flowering time in various transgenic lines

Box plots showing days to flowering in Ler WT, fwa-I and various transgenic lines in the fwa-I background (with 10 randomized replicates for each line). 
$1 \mathrm{~A}$
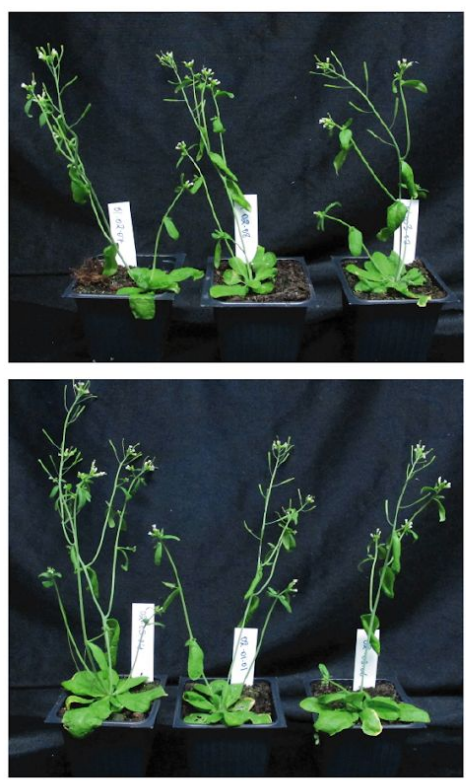

1B

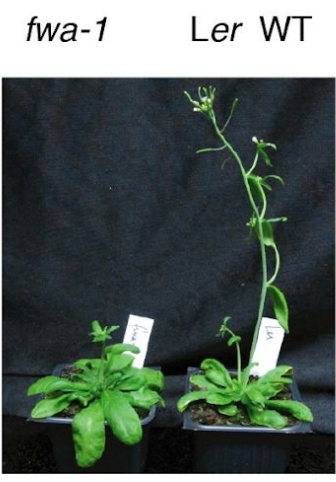

$2 \mathrm{~A}$
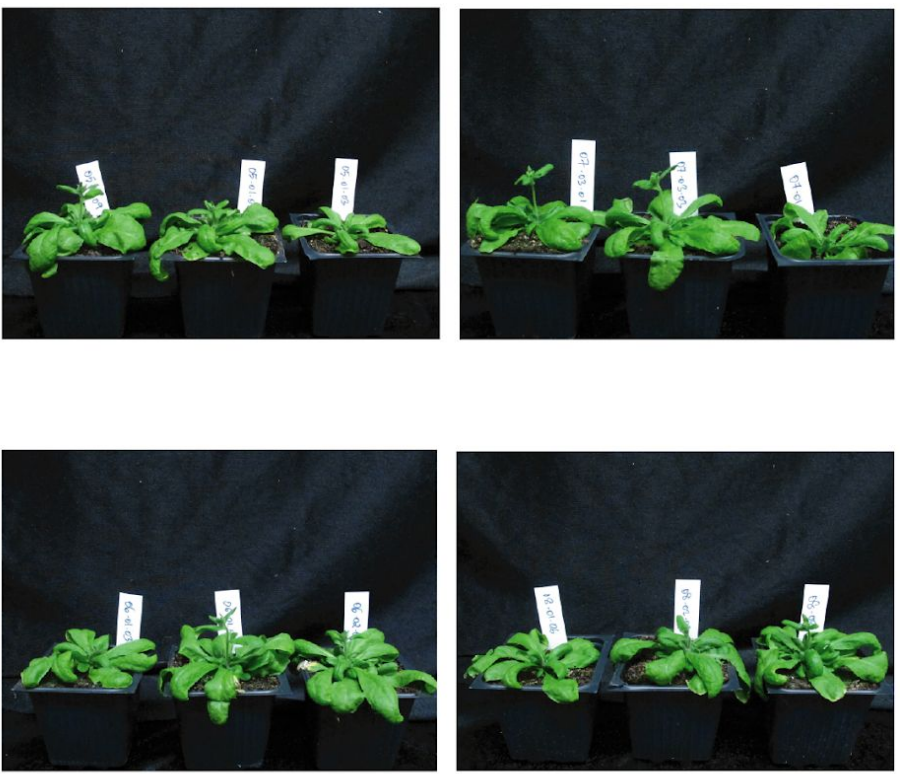

2B

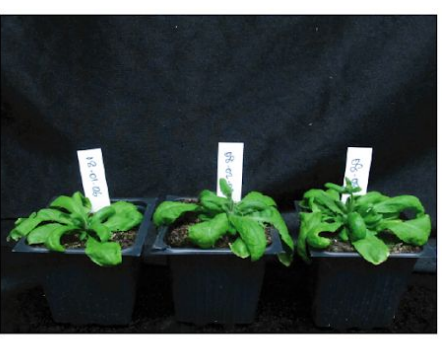

3B

Figure 3. Phenotypes of transgenic lines

Transgenic lines and control plants at 28 days after germination.

Consistent with these observations, FWA transcripts in lines IA and IB accumulated to levels much lower than in parental fwa-I, and more similar to those in early-flowering Ler wild-type plants, indicative of effective gene silencing (Figure 4). Similarly, lines in which flowering time was unaltered showed less reduction (lines $2 \mathrm{~A}, 2 \mathrm{~B}, 3 \mathrm{~A}$ ) or no reduction (line $3 \mathrm{~B}$ ) of $F W A$ mRNA accumulation (Figure 4). 


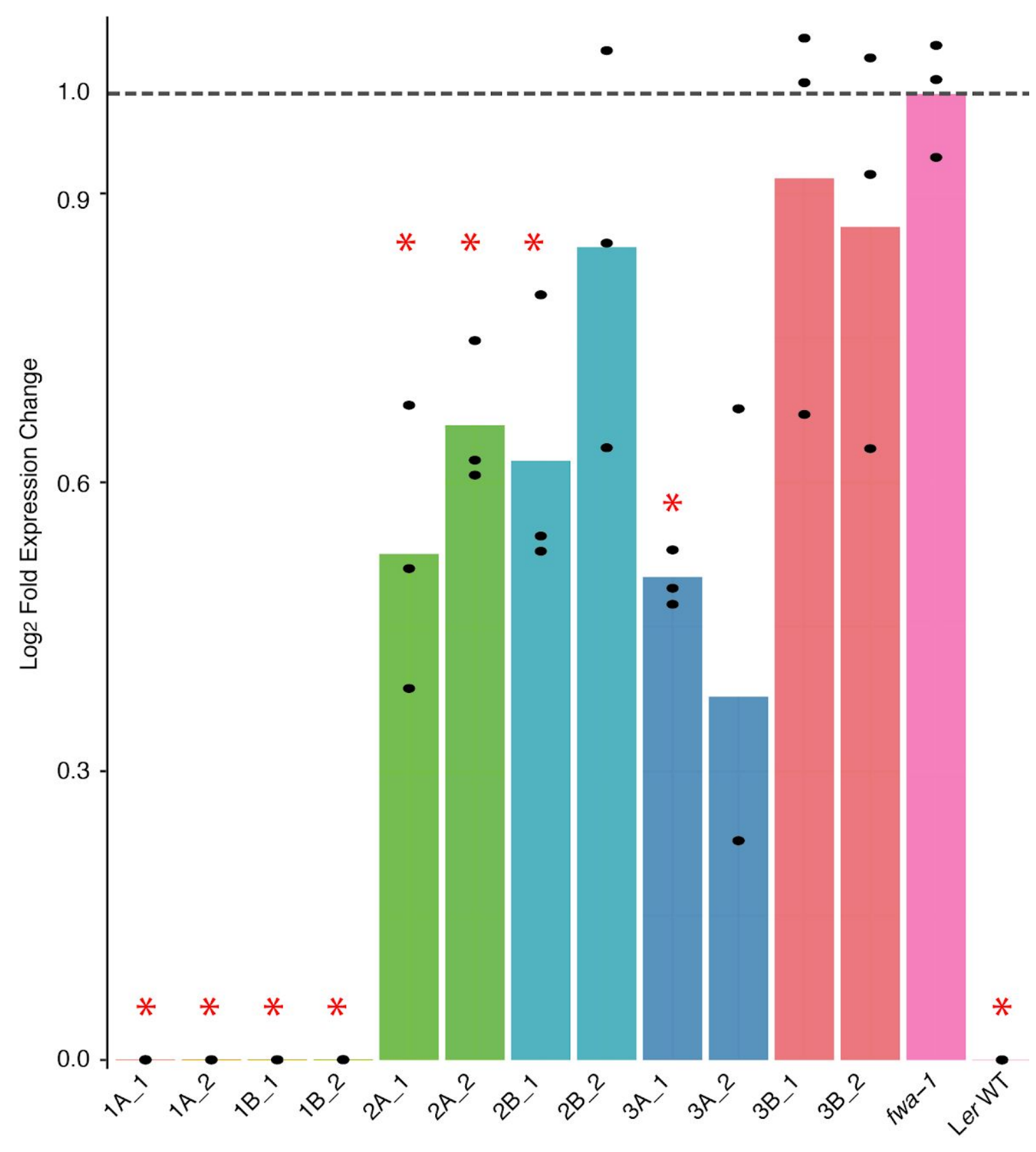

Plant Line

Figure 4. FWA mRNA accumulation in plants expressing IR-hairpins

Expression of FWA in LerWT, fwa-I and various transgenic lines carrying the hairpin-construct in sense and antisense orientations. Gene expression was measured by RT-qPCR. Bars show mean $\log _{2}$-fold change compared to fwa-l, with black dots representing pooled biological replicates. The red asterisks represent statistical significance ( $\mathrm{p}<0.05$ for a Student's t-test). 

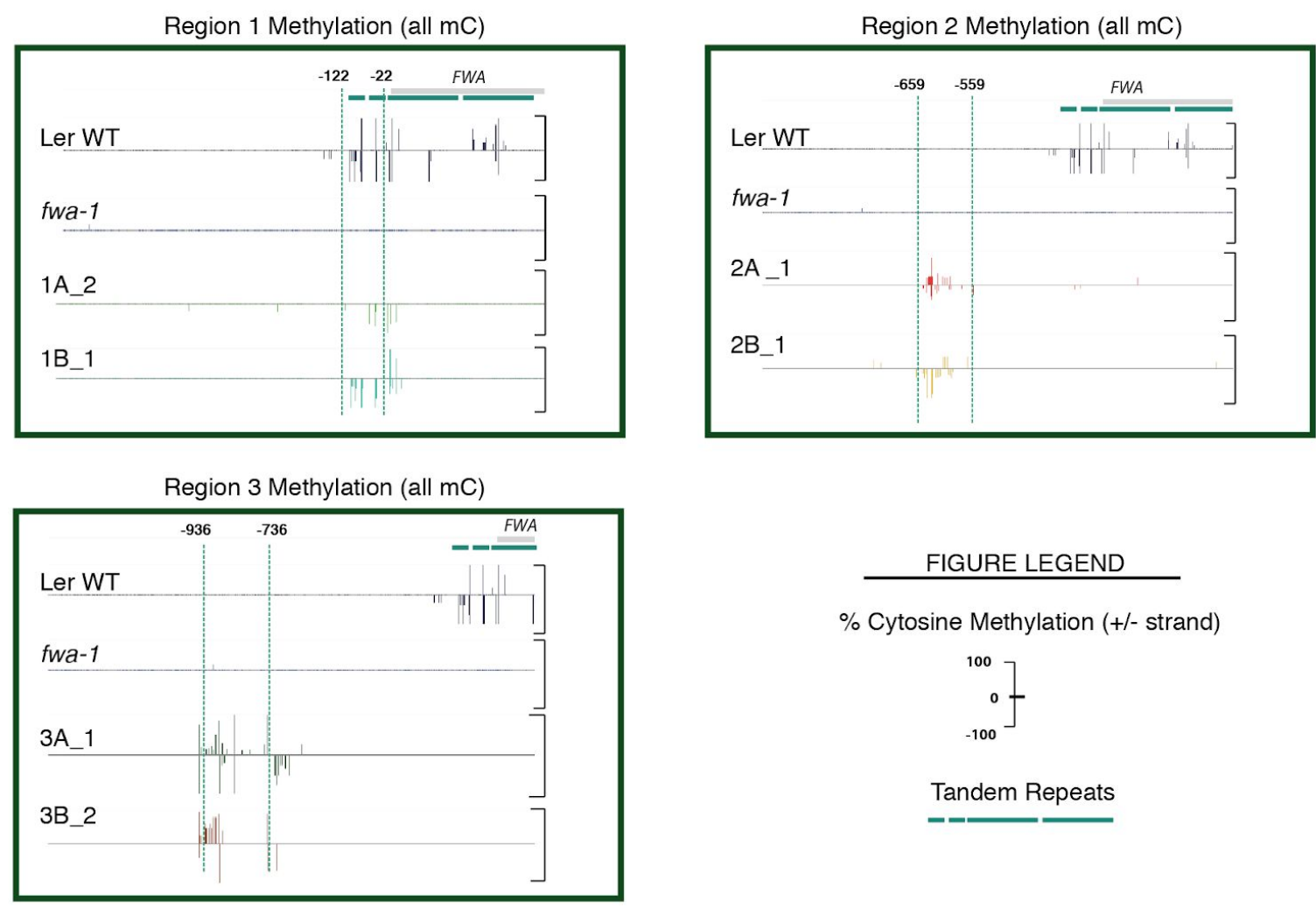

Figure 5. Methylation profile of targeted regions in transgenic lines

Hairpin-induced methylation for various transgenic lines. Each panel shows the methylation status of the targeted region in the Ler WT, fwa- $I$ and transgenic lines carrying the hairpin-construct in sense and antisense orientations. Green dotted lines demarcate the positions of each region. The vertical lines represent percentage methylation at every cytosine in both strands.

\section{Establishment of cytosine methylation at distal promoter regions}

As IR-hairpins derived from more distal regions of the FWA promoter did not induce sufficient FWA silencing to alter the timing of flowering, we asked whether cytosine methylation had been effectively established at the targeted sequences using whole-genome bisulfite sequencing. As shown in Figure 5, all six transgenes efficiently introduced methylation in the targeted regions to comparable levels. The effect of the transgene on methylation was stable over at least three generations, and indicates that cytosine methylation at this locus can be introduced 
not only at the SINE-derived repeat elements. We further conclude that methylation at more distal promoter elements was less effective in FWA silencing, which may be due to the distance from the transcription start site.

\section{DISCUSSION}

Cytosine methylation can be altered at many genomic regions under environmental stress. For example, phosphate starvation in rice and Arabidopsis thaliana results in the generation of hypermethylated differentially methylated regions (DMRs), many of which are found over transposable elements that are close to genes induced upon such nutrient starvation (2I). On the contrary, some studies report that stress-induced methylation and gene expression are not necessarily correlated with one another. In $A$. thaliana, most genomic regions exhibiting differential methylation under drought are not associated with expression changes of adjacent genes, with only 2 of 468 drought-response genes being linked to drought DMRs (22). These studies support the observation that methylation changes often flank protein-coding genes, yet only a few of them have been associated with a biological function. Several epialleles characterized in $A$. thaliana are marked by differential cytosine methylation in transposon-derived repetitive elements located proximal to the transcription start site (for example, SDC, (9) ; RMGI, (I0); HDG3, (23)). This pattern also holds true for the FWA locus, where SINE -derived tandem repeats that overlap with the transcription start site are heavily methylated and silence the gene.

Transcription is initiated in the proximity of DNA elements providing a platform for the recruitment and assembly of polymerase II-containing complexes. Their binding is modulated by enhancers and repressors, which themselves may recognize distal sequence elements. It is likely that cytosine methylation over elements of polymerase docking may directly or indirectly hinder effective protein recruitment. When located in regions distal to the transcription start site, methylation-based silencing may be achieved by the inhibition of transcriptional enhancers (24), and by altering short or long-distance chromatin interactions (25), thus affecting 
accessibility to the transcription machinery. Such a mechanism may possibly account for our observations, that methylation targeted in Regions 2 and 3, located more than 500 bp upstream of the transcription start site, only moderately impacts FWA transcript accumulation.

On examining publicly available data from the PlantDHS Browser (26), we observed that the I-kb promoter region immediately upstream of the FWA transcription start, including all three regions targeted for methylation, has a uniform state of chromatin accessibility (Figure 6), not only when in the silent wild-type state, but also in the unsilenced state in $d d m$-I mutants, which express FWA due to hypomethylation of the tandem repeats (27). Therefore, it may be expected that one would see a similar transcriptional readout upon modulating different regions within the same inaccessible sequence. At least in the FWA locus, this is not the case. Our results indicate that, while IR hairpin-induced methylation can be successfully introduced in the distal FWA promoter, this methylation can induce only moderate downregulation of FWA transcription, in contrast to very potent effects when methylating elements more proximal to the transcription start site.

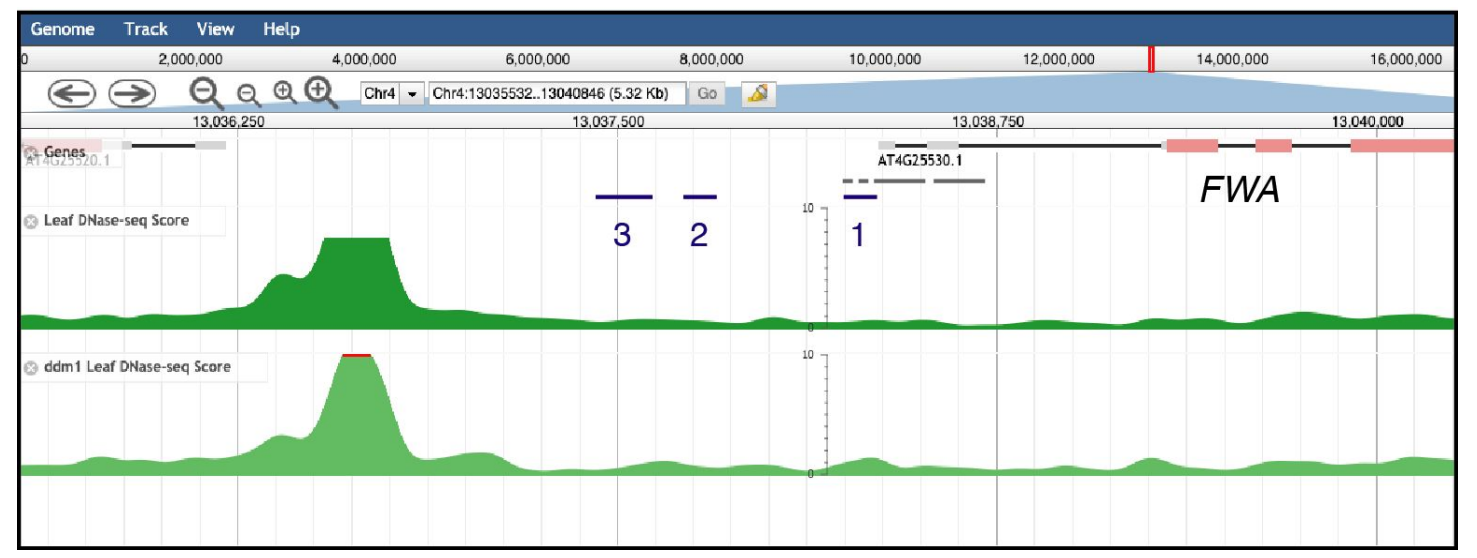

Figure 6. Chromatin conformation at the FWA locus

Screenshot from the PlantDHS browser (http://plantdhs.org/) showing the DNasel-seq profile of the FWA locus in leaf tissue of Col-0 wild-type (top panel) and ddm-I mutant (bottom panel) backgrounds. Grey bars below the FWA gene annotation indicate the tandem repeats; blue bars indicate the three regions targeted for methylation in this study. 
It will be interesting to examine the chromatin state and conformation of the FWA promoter in our transgenic lines, to understand whether they can explain the differential transcriptional downregulation that we observed.

This study was focused on the FWA locus; further investigation into differential promoter methylation at other genomic loci is required to dissect the mechanism behind methylation-dependent control on downstream transcription processes, and ultimately uncover its adaptive and biological function. 


\section{METHODS}

\section{Cloning of inverted-repeat hairpin (IR-Hairpin) constructs}

Three 100 bp - 200 bp regions in the FWA promoter were chosen for IR-hairpin construction (Table I and Supplementary Table SI), and cloned in two ways: All 'A' constructs carry sequences in sense orientation relative to the open-reading frame of the FWA locus in the hairpin 5' arm, while the 'B' constructs are reversed.

Hairpins, including flanking attB gateway sites were synthesised (GeneArt), PCR- amplified and transferred to pDONR207 (Invitrogen) using BP Clonase II . Recombinant entry clones were subjected to LR Clonase II reaction with the pJawohl-ACT2 destination vector (28) and introduced into E.coli DH5a (Invitrogen) cells by heat-shock transformation. Colonies carrying the hairpin construct in the correct orientation were verified by Sanger sequencing (oligonucleotide sequence provided in Supplementary Table SI).

Recombinant plasmids (listed in Supplementary Table S4) were introduced into Agrobacterium tumefaciens strain GV3I0I (PMP90RK) (29) by electro-transformation and grown in selective LB medium. Arabidopsis thaliana fwa-I mutants (16) were transformed with a floral dip protocol (30). Transgenic seeds were selected with I\% BASTA (Sigma-Aldrich).

\section{RT-qPCR}

Homozygous third-generation transformants $\left(\mathrm{T}_{3}\right)$ derived from three independent parent lines were sown on half-strength MS (Duchefa Biochemie) plates with sucrose and grown under long day (I6h light/8h dark) conditions at $23{ }^{\circ} \mathrm{C}$ for 10 days in a Percival chamber (Model CU -36L5, CLF Plant Climatics GmbH, Germany). For each line, 3 biological replicates of 20 pooled seedlings were subjected to RNA extraction (based on the LogSpin method, (3I)). cDNA synthesis was carried out with an equimolar concentration of an Oligo(dT)। 8 primer and an FWA gene-specific primer (Supplementary Table S2) using the RevertAid First Strand cDNA 
synthesis kit (Thermo Fisher Scientific), followed by qPCR of a I20bp region within the FWA gene body (primer sequences provided in Supplementary Table S2). The housekeeping gene ACTIN2 (At3GI8780) was used as a control gene for the experiment (primer sequences provided in Supplementary Table S2).

\section{Plant growth and flowering time analyses}

Seeds were sterilised by treatment with chlorine gas for 4 hours, followed by stratification in the dark at $4^{\circ} \mathrm{C}$ for 2 days in $0.1 \%$ agar. All plants were grown in controlled growth chambers at $23{ }^{\circ} \mathrm{C}$, long day conditions ( $16 \mathrm{~h}$ light/8h dark) with $65 \%$ relative humidity under 110 to 140 $\mu \mathrm{mol} \mathrm{m}^{-2} \mathrm{~s}^{-1}$ light provided by Philips GreenPower TLED modules (Philips Lighting GmbH, Hamburg, Germany) with a mixture of 2:I DR/W LB (deep red/white mixture with ca. I5\% blue) and W HB (white with ca. $25 \%$ blue), respectively and watered at 2-day intervals.

Ten plants belonging to each transgene were grown on soil in a randomized-block design, to reduce position effects in the growth chamber. Flowering time was recorded when the primary inflorescence meristem was approximately $\mathrm{Icm}$ in height.

\section{Bisulfite library preparation and sequencing}

Genomic DNA was isolated using the DNeasy Plant Mini Kit (Qiagen) from pools of twenty 10-day old seedlings grown on the same MS- Agar plates as seedlings used for RT-qPCR. I00ng of genomic DNA was used to prepare Bisulfite libraries with the TruSeq Nano kit (Illumina, San Diego, CA, USA) according to the manufacturer's instructions, with the modifications used in (I2). The libraries were sequenced with a paired-end mode, at I 25 million reads/library using an Illumina HiSeq3000 instrument.

\section{Processing of sequenced bisulfite libraries}

Raw sequencing reads were aligned using Bismark (default parameters; (32) and mapped to the A. thaliana (Landsberg erecta) reference genome. Bam files generated after deduplication of reads were processed for identifying methylated cytosines using pipelines as previously 
described in (33). Methylated cytosines in the FWA locus were loaded onto the EPIC-CoGe browser (34) for visualisation.

\section{Author contributions}

Study design: T.S., A.W., D.W. Experimental work and data analysis: T.S. Data interpretation: T.S., A.W., R.S., D.W. Drafting of initial manuscript: T.S. Editing and finalizing of manuscript: T.S., A.W., R.S., D.W.

\section{Acknowledgments}

We thank Claude Becker (Gregor Mendel Institute, Vienna) for providing access to the pipeline for DMR calling, and Isaac Rodriguez (Gregor Mendel Institute, Vienna) for his expertise. The pJawohl-ACT2 and pDONR207 plasmid vectors were kindly shared by José Gutierrez-Marcos (University of Warwick). This study was supported by Marie Sklodowska-Curie Fellowship 751204-H2020-MSCA-IF-2016 (A.W.), DFG (ERA-CAPS AUREATE), and the Max Planck Society. 


\section{REFERENCES}

I. Law JA, Jacobsen SE (2010) Establishing, maintaining and modifying DNA methylation patterns in plants and animals. Nat Rev Genet I I:204.

2. Johnson LM, et al. (2007) The SRA methyl-cytosine-binding domain links DNA and histone methylation. Curr Biol I7(4):379-384.

3. Kankel MW, et al. (2003) Arabidopsis METI cytosine methyltransferase mutants. Genetics I63(3): I I09-II 22.

4. Mathieu O, Reinders J, Caikovski M, Smathajitt C, Paszkowski J (2007) Transgenerational stability of the Arabidopsis epigenome is coordinated by $C G$ methylation. Cell I30(5):85 I-862.

5. Finnegan EJ, Dennis ES (1993) Isolation and identification by sequence homology of a putative cytosine methyltransferase from Arabidopsis thaliana. Nucleic Acids Res $2 \mathrm{I}(\mathrm{I} 0): 2383-2388$.

6. Furci L, et al. (2019) Identification and characterisation of hypomethylated DNA loci controlling quantitative resistance in Arabidopsis. Elife 8. doi: 10.7554/eLife.40655.

7. Slotkin RK, Martienssen R (2007) Transposable elements and the epigenetic regulation of the genome. Nat Rev Genet 8(4):272-285.

8. Quadrana L, et al. (2016) The Arabidopsis thaliana mobilome and its impact at the species level. Elife 5. doi:I0.7554/eLife. I57/6.

9. Henderson IR, Jacobsen SE (2008) Tandem repeats upstream of the Arabidopsis endogene SDC recruit non-CG DNA methylation and initiate siRNA spreading. Genes Dev 22(I2):I597-1606.

10. Fischer RL, Voinnet O, Navarro L (2013) Dynamics and biological relevance of DNA demethylation in Arabidopsis antibacterial defense. Proceedings of the. Available at: https://www.pnas.org/content/ I 10/6/2389.short.

II. Zicola J, Liu L, Tänzler P, Turck F (2019) Targeted DNA methylation represses two enhancers of FLOWERING LOCUS T in Arabidopsis thaliana. Nat Plants 5(3):300-307.

12. Wibowo A, et al. (2018) Partial maintenance of organ-specific epigenetic marks during plant asexual reproduction leads to heritable phenotypic variation. Proc Natl Acad Sci US A II5(39):E9I45-E9I52.

13. Lippman Z, et al. (2004) Role of transposable elements in heterochromatin and epigenetic control. Nature 430(6998):47I-476. 
14. Kinoshita T, et al. (2004) One-way control of FWA imprinting in Arabidopsis endosperm by DNA methylation. Science 303(5657):52 I-523.

15. Koornneef M, Hanhart CJ, van der Veen JH (199I) A genetic and physiological analysis of late flowering mutants in Arabidopsis thaliana. Mol Gen Genet 229(I):57-66.

16. Soppe WJ, et al. (2000) The late flowering phenotype of fwa mutants is caused by gain-of-function epigenetic alleles of a homeodomain gene. Mol Cell 6(4):79I-802.

17. Chan SW, Zhang X, Bernatavichute YV, Jacobsen SE (2006) Two-Step Recruitment of RNA-Directed DNA Methylation to Tandem Repeats. PLoS Biology 4(I I):e363.

18. Kinoshita Y, et al. (2007) Control of FWA gene silencing in Arabidopsis thaliana by SINE-related direct repeats. Plant J 49(I):38-45.

19. Mette MF, Aufsatz W, van der Winden J, Matzke MA, Matzke AJM (2000) Transcriptional silencing and promoter methylation triggered by double-stranded RNA. EMBO J 19(I9):5194-5201.

20. Johnson LM, et al. (20I4) SRA- and SET-domain-containing proteins link RNA polymerase $\checkmark$ occupancy to DNA methylation. Nature 507(7490): I 24-I 28.

2I. Secco D, et al. (20I5) Stress induced gene expression drives transient DNA methylation changes at adjacent repetitive elements. Elife 4. doi: 10.7554/eLife.09343.

22. Van Dooren TJM, et al. (2018) Mild drought induces phenotypic and DNA methylation plasticity but no transgenerational effects in Arabidopsis. bioRxiv.370320.

23. Pignatta D, Novitzky K, Satyaki PRV, Gehring M (2018) A variably imprinted epiallele impacts seed development. PLoS Genet I4(I I):el 007469.

24. O'Malley RC, et al. (2016) Cistrome and Epicistrome Features Shape the Regulatory DNA Landscape. Cell 166(6): 1598.

25. Crevillén P, Sonmez C, Wu Z, Dean C (2013) A gene loop containing the floral repressor FLC is disrupted in the early phase of vernalization. EMBO J 32(I): I40-I 48.

26. Zhang T, Marand AP, Jiang J (2016) PlantDHS: a database for DNase I hypersensitive sites in plants. Nucleic Acids Res 44(DI):DI I48-53.

27. Kakutani T (1997) Genetic characterization of late-flowering traits induced by DNA hypomethylation mutation in Arabidopsis thaliana. Plant J I2(6): |447-I45 I.

28. Wibowo A, et al. (2016) Hyperosmotic stress memory in Arabidopsis is mediated by distinct epigenetically labile sites in the genome and is restricted in the male germline by DNA glycosylase activity. Elife 5. doi: 10.7554/eLife. I 3546. 
29. Koncz C, Schell J (1986) The promoter of TL-DNA gene 5 controls the tissue-specific expression of chimaeric genes carried by a novel type of Agrobacterium binary vector. Mol Gen Genet 204(3):383-396.

30. Clough SJ, Bent AF (1998) Floral dip: a simplified method for Agrobacterium-mediated transformation of Arabidopsis thaliana. Plant J 16(6):735-743.

31. Yaffe H, et al. (20I2) LogSpin: a simple, economical and fast method for RNA isolation from infected or healthy plants and other eukaryotic tissues. BMC Res Notes 5:45.

32. Krueger F, Andrews SR (20I I) Bismark: a flexible aligner and methylation caller for Bisulfite-Seq applications. Bioinformatics 27(I I): I57I-I572.

33. Hagmann J, et al. (20I5) Century-scale methylome stability in a recently diverged Arabidopsis thaliana lineage. PLoS Genet I I(I):e 1004920.

34. Nelson ADL, Haug-Baltzell AK, Davey S, Gregory BD, Lyons E (2018) EPIC-CoGe: managing and analyzing genomic data. Bioinformatics 34(I 5):265 I-2653. 


\section{SUPPLEMENTARY DATA}

Table SI. Primer sequences for IR-hairpin cloning (FP forward primer, RP reverse primer)

\begin{tabular}{|c|c|}
\hline Sequence (5'-->3') & Purpose \\
\hline ATACGACTCACTATAGGGCGAA & $\begin{array}{c}\text { FP for amplification of attB-target sequence } \\
\text { from GeneART plasmid }\end{array}$ \\
\hline ATGTTAATGCAGCTGGCACGAC & $\begin{array}{c}\text { RP for amplification of attB-target sequence } \\
\text { from GeneART plasmid }\end{array}$ \\
\hline TTGATGCCTGGCAGTTCCCTA & $\begin{array}{c}\text { FP for Colony PCR of entry clones in } \\
\text { pDONR207 }\end{array}$ \\
\hline CATCAGAGATTTTGAGACACGGG & $\begin{array}{c}\text { RP for Colony PCR of entry clones in } \\
\text { pDONR207 }\end{array}$ \\
\hline GACAATGGTACCGGTATGGT & $\begin{array}{c}\text { ACT2 promoter Sequencing primer } \\
\text { (pJAW-ACT2 destination clones) }\end{array}$ \\
\hline TCGCGTTAACGCTAGCATGGATCTC & FP for pDONR207 entry clone sequencing \\
\hline GTAACATCAGAGATTTTGAGACAC & RP for pDONR207 entry clone sequencing \\
\hline GGGGACAAGTTTGTACAAAAAAGCAGGCT & attBL \\
\hline ACCCAGCTTTCTTGTACAAAGTGGTCCCC & attBR \\
\hline
\end{tabular}

Table S2. Primer sequence for RT-q-PCR analysis

\begin{tabular}{|c|l|}
\hline Sequence (5'-->3') & \multicolumn{1}{|c|}{ Purpose } \\
\hline CTTTGGTACCAGCGGAGA & cDNA synthesis primer for FWA \\
\hline TACATTGGGAGAAGTGGACTGT & FP for RT-qPCR FWA \\
\hline GGGAGGTTTTGTGACATTGTTG & RP for RT-qPCR FWA \\
\hline GCCATCCAAGCTGTTCTCTC & FP for RT-qPCR ACTIN2 \\
\hline GCTCGTAGTCAACAGCAACAA & RP for RT-qPCR ACTIN2 \\
\hline
\end{tabular}


Table S3. Regions targeted for methylation [Sequences in 5'-->3 orientation]

\begin{tabular}{|c|c|}
\hline Region IA & $\begin{array}{l}\text { GAGTTATGGGCCGAAGCCCATACATCTTTCCGTCGAGAATCTCAT } \\
\text { ATATTCTTTATCGAAGCCCATACATCTTTCCGTCGAGAATCTCATA } \\
\text { TATACCTTA }\end{array}$ \\
\hline Region IB & $\begin{array}{l}\text { TAAGGTATATATGAGATTCTCGACGGAAAGATGTATGGGCTTCGA } \\
\text { TAAAGAATAATGAGATTCTCGACGGAAAGATGTATGGGCTTCGGC } \\
\text { CCATAACTC }\end{array}$ \\
\hline Region 2A & $\begin{array}{l}\text { CСTCATCTGCGCTTATAAATAAGGCAAAGCAACTAGAAAAGATTA } \\
\text { AAACCAAAACCAAAACAAAAAACTAGTTAAGACCCTGATTTTGTTT } \\
\text { CATAGGTAC }\end{array}$ \\
\hline Region 2B & $\begin{array}{l}\text { GTACCTATGAAACAAAATCAGGGTCTTAACTAGTTTTTTGTTTTGG } \\
\text { TTTTGGTTTTAATCTTTTCTAGTTGCTTTGCCTTATTTATAAGCGCA } \\
\text { GATGAGG }\end{array}$ \\
\hline Region 3A & $\begin{array}{l}\text { ATTTTTTCTATCATTTCATATCATTGTAACTATAAATTTTCGTAAAT } \\
\text { AGACCTTTAGTGTTAATACAATAGATTTTTATTAATTTTATATCGGA } \\
\text { TTTTGTTTAAAAAAGAAAAACCATAGGATGGATGATGATTGGTACT } \\
\text { TATAAGATTGTAATTGGGTATTTTTGGATTGTTACCACCATTACAA } \\
\text { AGCTATTAACAGAG }\end{array}$ \\
\hline Region 3B & $\begin{array}{l}\text { CTCTGTTAATAGCTTTGTAATGGTGGTAACAATCCAAAAATACCCA } \\
\text { ATTACAATCTTATAAGTACCAATCATCATCCATCCTATGGTTTTTC } \\
\text { TTTTTTAAACAAAATCCGATATAAAATTAATAAAAATCTATTGTATT } \\
\text { AACACTAAAGGTCTATTTACGAAAATTTATAGTTACAATGATATGA } \\
\text { AATGATAGAAAAAAT }\end{array}$ \\
\hline
\end{tabular}


Table S4. Recombinant plasmids generated for this study

\begin{tabular}{|l|l|}
\hline Plasmid & Purpose \\
\hline PTS00 I & PJAW-ACT2 Destination vector targeting methylation in Region IA \\
\hline PTS002 & PJAW-ACT2 Destination vector targeting methylation in Region IB \\
\hline PTS003 & PJAW-ACT2 Destination vector targeting methylation in Region 2A \\
\hline PTS004 & PJAW-ACT2 Destination vector targeting methylation in Region 2B \\
\hline PTS005 & PJAW-ACT2 Destination vector targeting methylation in Region 3A \\
\hline PTS006 & PJAW-ACT2 Destination vector targeting methylation in Region 3B \\
\hline
\end{tabular}

